\title{
DIE AUGENHEILKUNDE
}

DES

IBN SINA

AUS DEM ARABISCHEN

ÜBERSETZT UND ERLÄUTERT

voN

J. HIRSCHBERG UND J. LIPPERT

LEIPZIG

VERLAG VON VEIT \& COMP.

1902 
Verlag von VEIT \& COMP. in Leipzig.

DIE MATHEMATISCHEN GRUNDLAGEN DER

MEDICINISCHEN STATISTIK

elementar dargestellt

von J. Hirschberg.

8. 1874. geh. 2 th 40 gF.

EINE WOCHE IN TUNIS.

Tagebuchblätter

von J. Hirschberg.

8. 1885 . geh. 2.

WÖRTERBUCH DER AUGENHEILKUNDE.

Von J. Hirschberg.

gr. 8. 1887. geh. 5 At.

VON NEW YORK NACH SAN FRANCISCO.

Tagebuchblätter

von J. Hirschberg.

S. 1888. geh. $4 \mathrm{~N}$.

HILFSWÖRTERBUCH

ZUM

ARISTOPHANES

von J. Hirschberg.

Erster Theil.

Die Acharner. Die Ritter. Die Wolken. Die Wespen. Der Frieden.

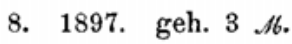

DIE MAGNET-OPERATION

IN DER

AUGENHEILKUNDE.

NACH EIGENEN ERFAHRUNGEN DARGESTELLT

von J. Hirschberg.

Zweite, vollständig neu bearbeitete Auflage.

Mit 30 Abbildungen im Text.

gr. 8. 1899. geh. 4 A6 40 gf. 


\section{DIE AUGENHEILKUNDE \\ DES}

\section{IB N SINA}

AUS DEM ARABISCHEN ÜBERSETZT UND ERLÄUTERT

voN

J. HIRSCHBERG UND J. LIPPERT

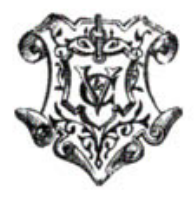

LEIPZIG

VERLAG VON VEIT \& COMP.

1902 
Druck von Metzger \& Wittig in Leipzig. 


\section{HERRN KARL EDUARD SACHAU ZUGEEIGNET}

VON DEN VERFASSERN 
